\title{
Prozess- und Zustandsüberwachung
}

\section{Sehr geehrte Leserinnen und Leser!}

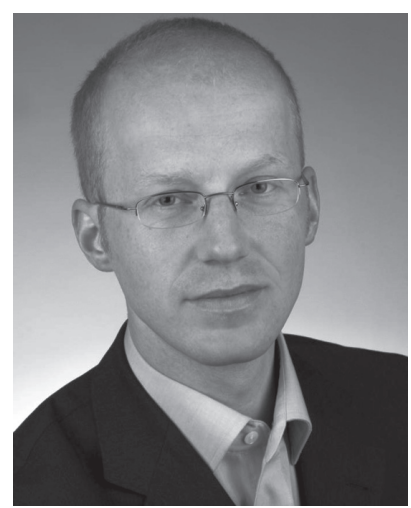

\section{Dr. Gerald Steiner}

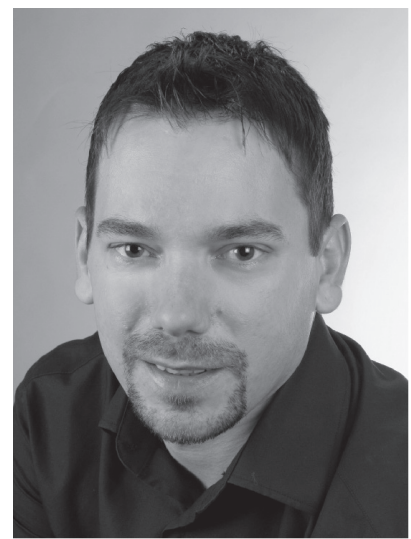

Dr. Daniel Watzenig

viel mehr Fehler- und Ausfallsmöglichkeiten. Je "mächtiger" und leistungsfähiger ein automatisierungstechnisches System ist, desto schwerwiegender können sich Fehlfunktionen auswirken: Enorme Schäden für Personen, Umwelt und auch finanzieller Natur können die Folge sein.

Die Prozess- und Zustandsüberwachung, der dieser Themenschwerpunkt der aktuellen e\&i-Ausgabe gewidmet ist, ermöglicht durch die kontinuierliche Verfolgung von industriellen Prozessen und den Zuständen komplexer Systeme erst die Einhaltung der genannten Ziele und die Vermeidung von kritischen Systemzuständen oder Fehlerfällen. Naturgemäß werden an die Überwachungssysteme spezielle Anforderungen wie hohe Ausfallsicherheit und gute Wiederholbarkeit der Messungen unter teilweise extremen und stark schwankenden Umgebungsbedingungen gestellt. Ein Eindruck von der Schärfe der Anforderungen ergibt sich bei der Durchsicht der folgenden Artikel, wo Sensoren und Methoden für den Einsatz zu Lande, zu Wasser, in der Luft und sogar im Weltall vorgestellt werden. Dabei sind dicke Eisschichten, brodelnde Flüssigkeiten, mehrere hundert Grade heiße korrosive Gase und hohe Geschwindigkeiten zu meistern.

Gerade die Kraftfahrzeugtechnik stellt höchste Anforderungen an die verwendeten Überwachungssysteme. Die Zuverlässigkeit und Genauigkeit in einem extremen Temperaturbereich, bei allen Wetterbedingungen und unter Schmutzeinwirkung steht an vorderster Stelle. Auf dieser Basis können Effizienz, Komfort und Sicherheit bei ständig steigender Leistungsfähigkeit der Verkehrsmittel gesichert werden.

Trotzdem treten immer wieder Systemfehler und -ausfälle auf, die zu Pannen, Rückholaktionen, aber auch zu Unfällen führen können. Dem kann durch eine umfangreichere präventive Zustandsüberüberwachung entgegengewirkt werden.

Die vorliegende e\&i-Ausgabe zur Prozess- und Zustandsüberwachung soll mit Schwerpunkt auf Österreich die intensiven und erfolgreichen wissenschaftlichen Aktivitäten in diesem Feld anhand ausgewählter Beiträge aufzeigen. Dabei sticht die Verwandtschaft der Aufgaben und Anforderungen von industriellen und automotiven Anwendungen ins Auge, was auch als Ansporn für gegenseitigen Wissenstransfer verstanden werden sollte. Weiters ist in den einzelnen Artikeln die offensichtlich große Bedeutung von internationalen Kooperationen eindrucksvoll belegt. So sind Autoren dieser Ausgabe neben österreichischen Universitäten und Forschungseinrichtungen an Universitäten in Deutschland, Belgien, Slowenien und den USA tätig. Europa und Nordamerika sind weltweit auch nach wie vor die mit Abstand wichtigsten Märkte für Prozessautomatisierung. Die vorgestellten Forschungsarbeiten sind durchwegs von großer Praxisrelevanz und Aktualität. Das wird durch die Beteiligung führender Industrieunternehmen und öffentlicher Fördergeber und im Beitrag von Prof. F. Mesch zusätzlich durch Zusammenhänge mit geltender Normung unterstrichen.

In diesem Sinne danken wir den Autoren für die spannenden Beiträge, freuen uns auf den zukünftigen Einsatz der neuartigen Methoden zur Prozess- und Zustandsüberwachung und wünschen Ihnen, liebe Leserinnen und Leser, eine angeregte Lektüre!

\section{Dr. Gerald Steiner}

Anton Paar $\mathrm{GmbH}$,

Institut für Elektrische Messtechnik und Messsignalverarbeitung, Technische Universität Graz

\section{Dr. Daniel Watzenig}

Vehicle Electrics and Electronics Group,

Virtual Vehicle Competence Center,

Institut für Elektrische Messtechnik und Messsignalverarbeitung, Technische Universität Graz 